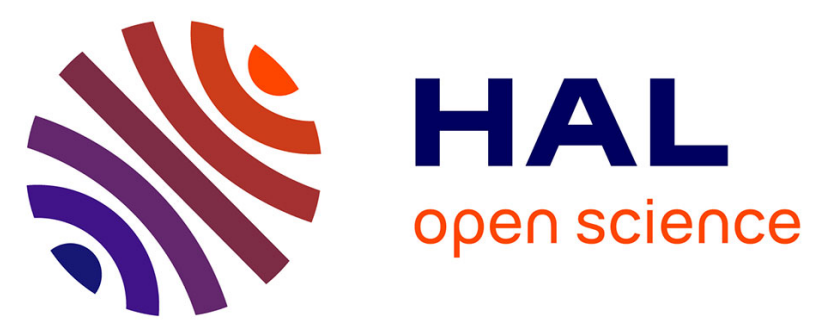

\title{
Acceleration statistics of inertial particles in turbulent flow
}

\author{
Muhammad Nauman Qureshi, Unai Arrieta, Christophe Baudet, Alain H. \\ Cartellier, Yves Gagne, Mickaël Bourgoin
}

\section{- To cite this version:}

Muhammad Nauman Qureshi, Unai Arrieta, Christophe Baudet, Alain H. Cartellier, Yves Gagne, et al.. Acceleration statistics of inertial particles in turbulent flow. The European Physical Journal B: Condensed Matter and Complex Systems, 2008, 66 (4), pp.531-536. 10.1140/epjb/e2008-00460-x . hal-00492267

\section{HAL Id: hal-00492267 https://hal.science/hal-00492267}

Submitted on 16 Jun 2010

HAL is a multi-disciplinary open access archive for the deposit and dissemination of scientific research documents, whether they are published or not. The documents may come from teaching and research institutions in France or abroad, or from public or private research centers.
L'archive ouverte pluridisciplinaire HAL, est destinée au dépôt et à la diffusion de documents scientifiques de niveau recherche, publiés ou non, émanant des établissements d'enseignement et de recherche français ou étrangers, des laboratoires publics ou privés. 


\title{
Acceleration statistics of inertial particles in turbulent flow
}

\author{
Nauman M. Qureshi, Unai Arrieta, Christophe Baudet, Alain Cartellier, Yves Gagne, Mickaël \\ Bourgoin \\ Laboratoire des Écoulements Géophysiques et Industriels, CNRS/UJF/INPG UMR5519, BP53, 38041 Grenoble, France \\ e-mail: mickael. bourgoin@hmg.inpg.fr
}

The date of receipt and acceptance will be inserted by the editor

\begin{abstract}
Turbulent transport of material inclusions plays an important role in many natural and industrial situations. Being able to accurately model and predict the dynamics of dispersed particles transported by a turbulent carrier flow, remains a challenge. One critical and difficult point is to develop models which correctly describe the dynamics of particles over a wide range of sizes and density. Our measurements show that acceleration statistics of particles dispersed in a turbulent flow do exhibit specific, and so far unpredicted, size and density effects and that they preserve an extremely robust turbulent signature with lognormal fluctuations, regardless of particles size and density. This has important consequences in terms of modeling for the turbulent transport of dispersed inclusions.
\end{abstract}

\section{Introduction}

Turbulent transport of material inclusions plays an important role in many natural and industrial processes. In marine ecosystem for instance, interactions between the turbulent sea and the gametes of marine animals is determinant for the efficiency of the reproduction and the spreading of the species (1). Industrial stakes concern mixing and combustion, while environmental issues such as pollutants dispersion (2) and pollenization (3) are more than ever crucial. In spite of decades of active theoretical, numerical and experimenal research, being able to accurately model and predict the dynamics of dispersed inclusions transported by a turbulent flow, still remains a challenge with important scientific, environmental and economical issues.

One critical and difficult point is to find a model which would correctly describe particles dynamics over a wide range of sizes and densities. The dynamics of particles transported by a turbulent flow is indeed known to be affected by size and density effects. If particles are neutrally buoyant and small (namely with their diameter $D$ much smaller than the smallest turbulent eddies, at the energy dissipation scale $\eta$ of the flow), they behave as fluid tracers and their dynamics reflects fluid particles dynamics. This property is commonly used to characterize single phase flows from tracer particles imaging techniques (5) (such as Particle Image Velocimetry, Laser Doppler Velocimetry, Particle Tracking Velocimetry, etc.). However, when particles density is larger or smaller than the surrounding fluid and/or when particles size becomes comparable to turbulent eddies', their dynamics deviates from that of fluid particles and tends to be affected by so called inertial effects. Among them some can be qualitatively described in terms of particles interaction with turbulent eddies. For instance, centrifugal forces are generally expected to cluster heavier particles in low vorticity regions (and lighter in high vorticity ones), an effect known as preferential concentration (6). However, from a quantitative point of view no reliable model has emerged yet to describe and predict accurately the statistical properties of particles advected by a turbulent flow. For instance we are unable today to

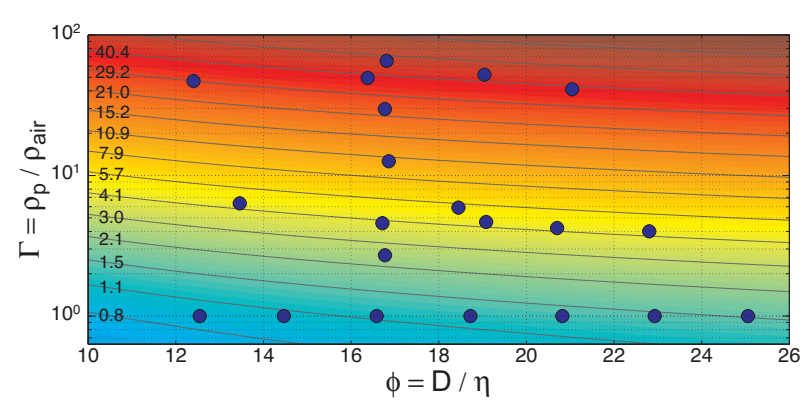

Fig. 1 Particle classes considered in the present study, described in the $(\phi, \Gamma)$ phase space. $\phi=D / \eta$ is the ratio of particles diameter to kolmogorov scale and $\Gamma=\rho_{\mathrm{p}} / \rho_{\text {air }}$ is the particle to fluid density ratio. The countour lines indicate an estimation of the particles Stokes number $S t=\tau_{p} / \tau_{D}$, where the respone time $\tau_{p}$ of the particle has been corrected for added mass and for finite Reynolds number effects according to Schiller and Nauman (4) and $\tau_{D}$ is the flow eddy turnover time at the scale of the particle diameter $D$. 


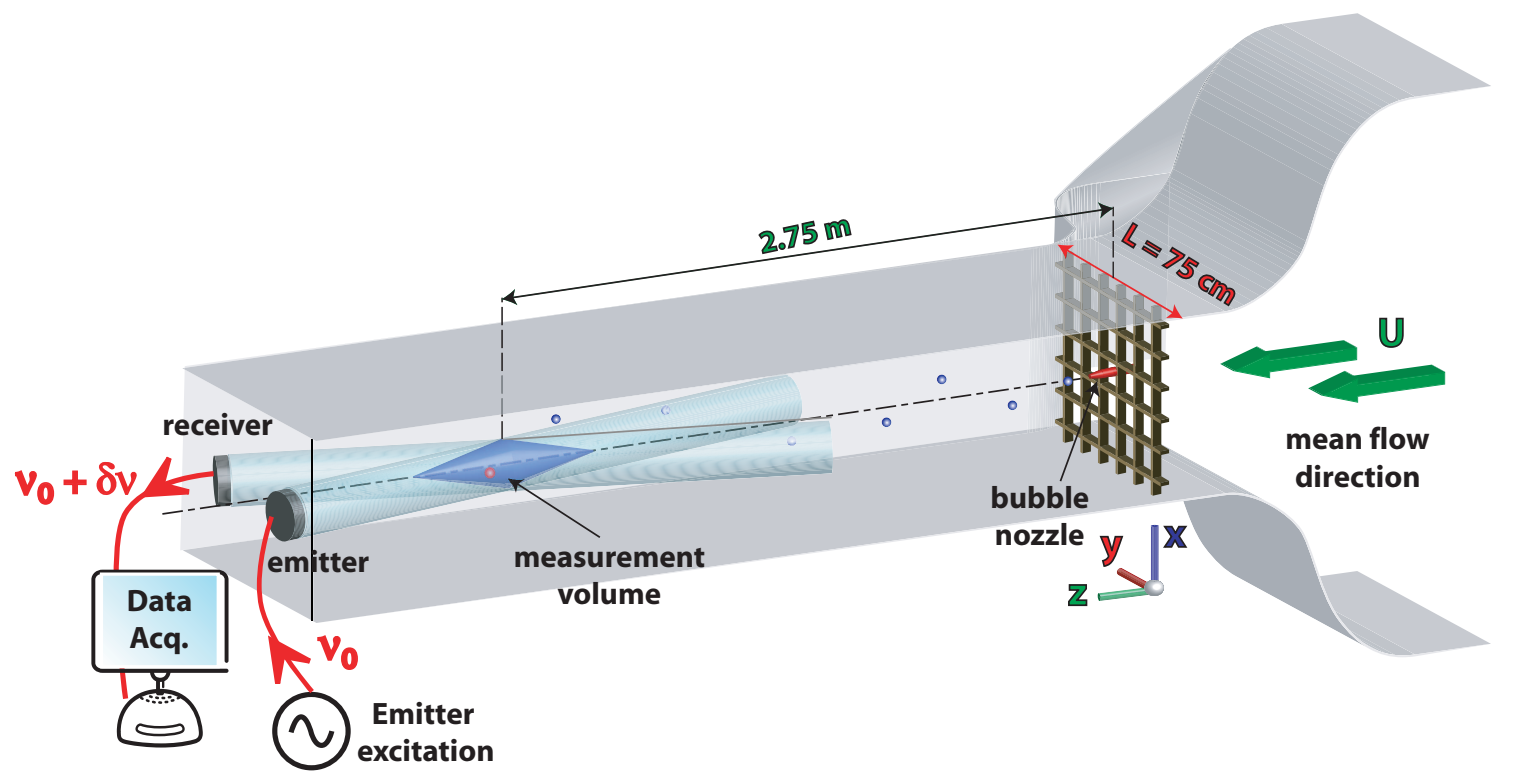

Fig. 2 Turbulence is generated downstream a grid in a wind tunnel. The measurement volume is located $2.75 \mathrm{~m}$ downstream the grid, in a region where turbulence is fully developped, homogeneous and isotropic. Particles are injected individually at the grid with a bubble nozzle and tracked individually by Doppler velocimetry. An ultrasonic plane wave of frequency $\nu_{0}$ is emitted by a transducer and a receiver records the wave scattered by a moving particle with velocity $\mathbf{v}$ in the measurement volume (which is the intersection of the emmitting and receiving acoustical beams, represented by the light cloroed tubes on the sketch). The component $v_{\|}$of the particle velocity along the scattering vector is directly given by the Doppler shift $\delta_{\nu}$ of the frequency of the recorded scattered wave. In the present experiment, transuducers are arranged in order to measure the sreamwise component $v_{z}$ of particles velocity.

predict correctly the dynamics of water droplets in a cloud $(7 ; 8)$, what considerably limits our understanding of rain mechanisms. Actually, even writing an appropriate equation of motion for a particle transported by a turbulent flow remains a theoretical challenge which has only been approached in some limit cases, assuming generally point like particles $(9 ; 10 ; 11 ; 12 ; 13 ; 14 ; 15)$. The range of validity of such models for real particles, with finite size and finite density, as well as the minimal forcing terms for a model to be pertinent remain unclear. In this context, the study of particles Lagrangian acceleration, which directly reflects the turbulent forcing exerted on the particles, has been particularly active in the past decade $(16 ; 17 ; 18 ; 19 ; 20 ; 21 ; 22)$. It is now well known that fluid particles exhibit highly nongaussian acceleration probability density function (PDF) with broad probability tails at high acceleration events (16). Direct numerical simulations in the limit of highly inertial point particles suggest that acceleration PDF tends to narrow and to gaussianize as particles inertia increases. On the experimental side, studies in the limit of very small $(D \ll \eta)$ and highly inertial particles $\left(10^{3}\right.$ denser than the carrier flow) seem to confirm this trend $(18 ; 19)$. However, recent measurements with finite size $(D \gtrsim \eta)$ weakly inertial particles (up to 2.5 times denser than the carrier flow) do not show any significant change of acceleration PDF with inertia $(21 ; 22)$.

In the present article, we report an exhaustive experimental investigation of material particles acceleration in a turbulent air flow, over a wide range of sizes and densities. In particular we consider the so far unexplored regime of finite size $(D>\eta)$ and highly inertial particles (much denser than the carrier fluid). We consider a turbulent carrier flow generated downstream a grid in a wind tunnel experiment.

The article is organized as follows : in section 2 we present the particles parameter space, which describes the range of particles size and density we have effectively explored ; in section 3 we describe the wind tunnel setup and the characteristic scales of the carrier turbulent field ; in section 4 we present the results obtained concerning particles acceleration statistics ; we finally propose a discussion of these results in section 5 before concluding in section 6 .

\section{Particles Parameter Space}

The aim of the present work is to explore acceleration statistics of inertial particles over a wide range of sizes and densities. In order to do so, we have developped a versatile particle generator which produces in situ adjustable spherical soap bubbles. Their density can be adjusted from neutrally buoyant to about 70 times denser than air. Neutrally buoyant particles are obtained by inflating the bubbles with Helium in order to compensate the weight of the soap film. Heavier particles are inflated with air, and their density is essentially controled by the 


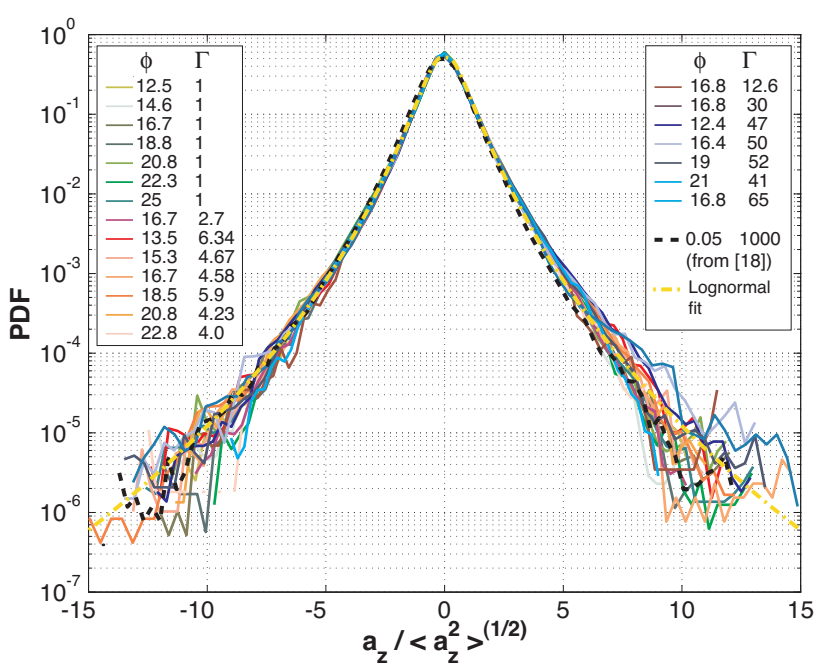

Fig. 3 Normalized component acceleration probability density function (PDF) of material particles transported in a turbulent air flow. Dashed line corresponds to the measurement by Ayyalasomayajula et al. (18) for water droplets (with size $\phi \sim 5 \times 10^{-2}$ and $\left.\Gamma \sim 1000\right)$. To help the comparison with previous and future studies the dot-dashed line shows a fit by the relation $\mathcal{P}(x)=\frac{e^{3 s^{2} / 2}}{4 \sqrt{3}}\left[1-\operatorname{erf}\left(\frac{\ln (|x / \sqrt{3}|)+2 s^{2}}{\sqrt{2} s}\right)\right]$ associated to a lognormal distribution of the acceleration amplitude $(23 ; 20)$; best fit is found for $s \sim 0.62$, corresponding to a distribution flatness $\mathcal{F}=\frac{9}{5} e^{4 s^{2}} \sim 8.3$.

thickness of the soap film. In all cases the bubbles Weber number remains extremely small, so that they do not deform and behave as rigid spheres. The seeding density is extremely low (particles are injected individually) so that particles can be considered as isolated and do not backreact on the carrier flow. Particles are therefore only characterized by two parameters : the ratio $\phi$ of their diameter $D$ to the dissipative scale of the flow $(\phi=D / \eta)$ and the ratio $\Gamma$ of their density $\rho_{\mathrm{p}}$ to the carrier fluid (air in our case) density $\rho_{\text {air }}\left(\Gamma=\rho_{\mathrm{p}} / \rho_{\text {air }}\right)$. Figure 1 summarizes all the particle classes (in the $(\phi, \Gamma)$ parameter space) that we have investigated. As far as we know this represents the most exhaustive exploration over such a wide range of particles sizes and densities.

\section{Experimental Setup}

Our experiment runs in a large wind tunnel (figure 2) with a measurement section of $0.75 \mathrm{~m} \times 0.75 \mathrm{~m}$ where the turbulence is generated downstream a grid with a mesh size of $6 \mathrm{~cm}$ and reproduces almost ideal isotropic turbulence. The results reported here were obtained with a mean velocity of the fluid $U=15 \mathrm{~m} \cdot \mathrm{s}^{-1}$ and a turbulence level $u_{r m s} / U \simeq 3 \%$. The corresponding Reynolds number, based on Taylor microscale, is of the order of $R_{\lambda}=160$. The dissipation scale $\eta=\left(\nu^{3} / \epsilon\right)^{1 / 4}$ is $240 \mu \mathrm{m}$ (where $\nu$ is the kinematic viscosity of air and $\epsilon$ the tur- bulent energy dissipation rate per unit mass) and the energy injection scale $L$ is $6 \mathrm{~cm}$. The carrier turbulent field has been characterized using classical hotwire anemometry. In particular, using a Taylor hypothesis, this gives access to the Eulerian correlation function and to the turbulent velocity gradients from which we estimate the integral scale $L$ and the dissipation rate $\epsilon$ respectively.

Particles are individually tracked using 1D Lagrangian acoustic Doppler velocimetry (see figure 2), where the instantaneous Doppler shift is obtained by a maximum of likelyhood parametric algorithm $(24 ; 25 ; 26)$. We measure the streamwise velocity component $v_{z}$ of the particles as they are tracked along their trajectories. The streamwiase acceleration component $a_{z}$ is obtained by differentiating the velocity using a convolution with a differentiated gaussian kernel (27). Each particle is tracked during approximately $50 \mathrm{~ms}$, which corresponds to several dissipation time scales $\tau_{\eta}=(\nu / \epsilon)^{1 / 2} \simeq 3.8 \mathrm{~ms}$. For each point in the $(\phi, \Gamma)$ parameter space we record about 4000 tracks at a samplimg rate of $32768 \mathrm{~Hz}$, giving more than $10^{6}$ data points per set.

\section{Acceleration Statistics}

Figure 3 represents acceleration PDFs normalized to variance one, $\left(a_{z} /\left\langle a_{z}^{2}\right\rangle^{1 / 2}\right)$, for all studied particles. Remarkably we find that all the PDFs almost collapse onto a single curve, indicating that, over the $(\phi, \Gamma)$ range considered, normalized acceleration statistics depends significantly neither on particle size, nor on particle density. In particular, contrary to predictions from point particle models, we do not observe any trend to gaussianization, even for particles with Stokes number $S t$ (see caption of figure 1 for the definition of $S t$ ) as large as 40 ; within statistical errorbars, we do not observe any systematic trend of acceleration flatness, which keeps a constant value $\mathcal{F} \simeq 8.3$ with $10 \%$ standard deviation among the different particle classes. This result extends to the case of heavy particles the robustness of acceleration PDF which we have previously reported for neutrally buoyant particles (20). It is also qualitatively consistent with recent measurements, in water von Kármán flows with weakly inertial particles $(28 ; 21 ; 22)$, although an unambiguous quantitative comparison with those results cannot be made due to experimental specificities (grid turbulence and von Kármán flows have very different large scale anisotropy and confinement characteristics which are known to affect particles Lagrangian dynamics $(29 ; 30))$. A quantitative comparison is however possible with recent measurements obtained by Z. Warhaft's group at Cornell University in a turbulent air flow with comparable characteristics to ours (both are windtunnel experiments with similar isotropy levels), although at a slightly higher Reynolds number $\left(R_{\lambda} \sim 250\right)$. Using high speed optical tracking, they have measured acceleration statistics of sub-kolmogorov water droplets $\left(\phi \sim 5 \times 10^{-2}\right.$ 


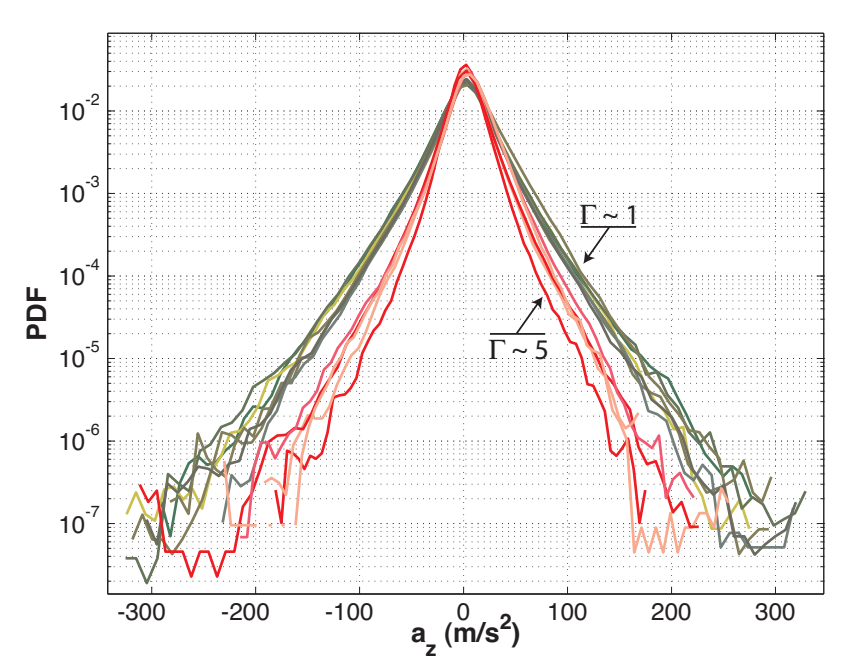

Fig. 4 Non normalized component acceleration PDF for $\Gamma \sim 1$ (outer bundle, green tone) and $\Gamma \sim 5$ (inner bundle, red tone) particles and various values of $\phi$.

; $\Gamma \sim 1000 ; S t \sim 0.1$ ), a regime of size which we cannot reach at present due to technical limitations of our bubble generator. We have superimposed on figure 3 the normalized acceleration PDF from their measurements (black dashed line). We find it to be almost undistinguishable from our measurements with larger particles. This suggests that acceleration of inertial particles as small as $\phi \sim 5 \times 10^{-2}$ already exhibit the same robust statistical signature as finite size particles ; point particle models may therefore only apply to even smaller particles (19).

For a deeper insight into specific effects associated to particles size and density, one has to investigate acceleration variance. Since the statistics of acceleration normalized to variance one is found essentially independent of particles size and density, an effect of those parameters can indeed only be expected to affect acceleration variance itself $\left\langle a_{z}^{2}\right\rangle$. Figure 4 represents the true acceleration PDF (not normalized to variance one) for neutrally buoyant $(\Gamma \sim 1)$ and heavy $(\Gamma \sim 5)$ particles, with varying sizes. It appears that heavy particles have a much narrower and peaked PDF, indicating a global decrease of acceleration variance with increasing density. The spreading of the curves for a given density indicates that size also influences particles acceleration variance.

A closer analysis of acceleration variance shows a non-trivial dependence with size and density. We report in figure 5 the acceleration variance for all the measurements we have performed (in the following, we consider the dimensionless acceleration variance normalized according to Heisenberg-Yaglom's scaling : $A_{0}(\phi, \Gamma)=$ $\left.\left\langle a_{z}^{2}\right\rangle \epsilon^{-3 / 2} \nu^{1 / 2}\right)$. The mapping of the $(\phi, \Gamma)$ parameter space that we have been able to explore, allows us to infer a rough interpolation of the evolution of $A_{0}(\phi, \Gamma)$, represented by the surface in figure 5 . Though only the

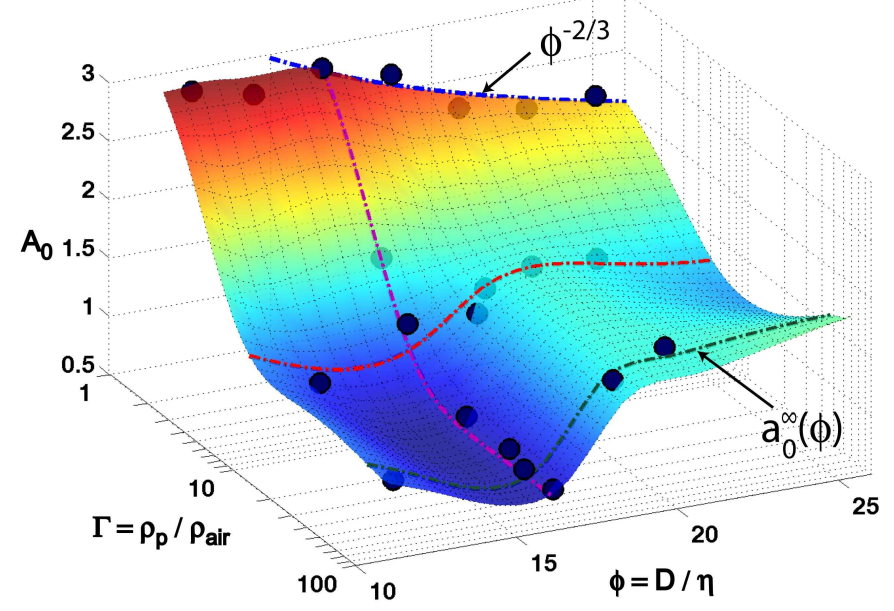

Fig. 5 Acceleration variance for all particles studied in the $(\phi, \Gamma)$ parameter space (dots). The surface represents a rough interpolation based on the available measurements (a biharmonic spline interpolation (31) has been used). The dot dashed lines materialize the interpolation along the best resolved constant $\phi$-lines and $\Gamma$-lines. For the $\Gamma=1$ set, the dot-dashed line coincide with a $\phi^{-2 / 3}$ decay for $\phi \gtrsim 15$ $(20 ; 16)$.

coarse tendency of this interpolation is relevant, several important qualitative features can still be observed. If we consider the effect of an increasing density at a fixed particle size (along constant $\phi$-lines), $A_{0}$ is always found to decrease and to saturate to a finite limit (noted $a_{0}^{\infty}(\phi)$ ) for the largest densities. This is better quantified in a $2 \mathrm{D}$ projection (figure 6 ). The $\phi \simeq 16.5$ set of measurements (which is our most complete set of density effects at fixed size) shows that the evolution of acceleration variance with density exhibits two different regimes (see inset in figure 6): (i) for low densities $A_{0}(\phi, \Gamma)=a_{0}(\phi) \Gamma^{-\alpha}$ $\left(a_{0}(\phi)\right.$ corresponds to size effects for the neutrally buoyant case which we have previously studied in (20)) with $\alpha \simeq 0.6$ (ii) for large densities $A_{0}$ saturates to a finite limit, here $a_{0}^{\infty}(\phi \simeq 16.5) \simeq 0.7$; the transition between these two regimes occurs for a characteristic density ratio $\Gamma^{*} \sim 10$. Although we don't have as many systematic measurements for varying densities for other values of $\phi$, the few available points are compatible with the existence of such two regimes (figure 6 ), with a size dependent saturation value $a_{0}^{\infty}(\phi)$ and very likely a size dependent transition density ratio $\Gamma^{*}(\phi)$.

If we focus now on size effects at fixed particle density (along constant $\Gamma$-lines), as seen on figure 5 , the scenario is rather complex: depending on the density ratio $\Gamma, A_{0}$ can either decrease or increase with particles size. For neutrally buoyant particles $(\Gamma=1)$ larger than about $\phi \sim 15, A_{0}$ decreases monotically as $a_{0}(\phi) \sim \phi^{-2 / 3}(20$; $16)$. We have shown in a previous study (20) that this is the expected scaling for inertial range sized particles when the main forcing simply comes from the spatial pressure differences of the unperturbed flow around 


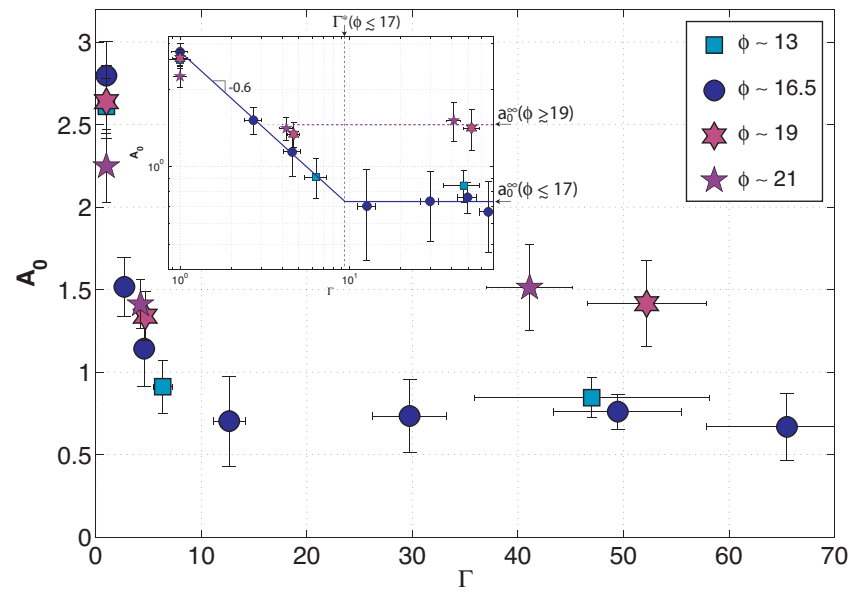

Fig. 6 2D-Projection on the $\left(\Gamma-A_{0}\right)$ plane of measurements data points on figure 5 , showing the evolution of acceleration variance with density ratio $\Gamma$ for different particle sizes ( $\phi \simeq 16.5$ set (circles) is the best resolved). The inset shows the same data in a log-log plot. Errorbars are mostly due to experimental uncertainties in the determination of particles size and density.

the particle. As we move to larger density ratios we then observe a continuous transition toward a drastically changed size dependence where $a_{0}^{\infty}(\phi)$ exhibit a sharp increase for sizes around $17<\phi<19$. Outside this transition region (i.e. for $\phi \lesssim 17$ and $\phi \gtrsim 19$ ), $a_{0}^{\infty}$ doesn't exhibit significant dependence on $\phi$ (at least in the accessible range of sizes) as also seen on figure 6 , where we have $a_{0}^{\infty}(13) \simeq a_{0}^{\infty}(16.5)$ and $a_{0}^{\infty}(19) \simeq a_{0}^{\infty}(21)$.

\section{Discussion}

Our measurements have important consequences in terms of the development of accurate models for the turbulent transport of finite sized inertial particles. The robustness of normalized acceleration PDF, regardless of particles size and density, and the non trivial size and density dependence of acceleration variance impose strong constraints on the statistical properties of the forcing terms to be included in the equation of motion of particles. For instance models based on Maxey \& Riley equation $(9 ; 10)$ for inertial point particles are contradictory with our observations as they predict a continuous trend of acceleration PDF to gaussianity as well as a monotonic decrease of acceleration variance $(13 ; 21 ; 32)$ both mostly consequence of a filtering effect due to increasing particles response time as inertia (only parametrized in such models by a Stokes number) increases. Our findings therefore show that turbulent transport of finite sized inertial particles cannot be simply described in terms of Stokes number and filtering effects only. The concrete statistical constraints that we have identified give a first clear experimental diagnose of point-particle models limitations (already analyzed theoretically by Lumley in $1978(33)$ ), and of the requirement for density and finite size effects to be simultaneously and specifically modeled.

With this goal, finally, we briefly discuss a simple qualitative phenomenology for the observed trends of acceleration variance based on the idea that particles tend to reside longer in the quietest regions of the flow. This intuitive statement can be related to the tendency of inertial particles to preferentially concentrate in low vorticity and low acceleration regions of the carrier turbulent field along which they are then advected folowing a sweep-stick scenario $(34 ; 35)$. In this context, the decrease of particles acceleration variance naturally results from the under-sampling of intense regions of the carrier flow. Then, a possible interpretation for the increase of acceleration variance with particle size observed for high density ratios can be that while small enough particles can indeed be expected to efficiently hide in the quietest regions of the flow, when particles become larger than the typical size $L^{*}$ of these regions, the sampling effect is damped as particles start experiencing again the influence of more active regions of the turbulent field. Though the existence of such a typical scale is still controversial, recent measurements of preferential concentration suggest that the quiet sticking regions might have a characteristic size $L^{*}$ in the range $10-20 \eta$ (36) consistent with the $17 \lesssim \phi \lesssim 19$ range for which we observe the sudden increase of $a_{0}^{\infty}(\phi)$.

Our measurements of inertial effects for finite size particles can therefore be consistently interpreted as a dominant role of sampling rather than filtering, contrary to the point particle case $(13 ; 32)$. The dominant role of sampling is also consistent with the fact that velocity statistics of our particles (not shown here) are found identical to that of the carrier flow (obtained from classical hotwire anemometry), a feature also shown numerically in the context of sweep-stick mechanism (35) but which is at odds from usual predictions based for instance on the Tchen-Hinze theory or extensions of this theory (37).

\section{Conclusion}

To summarize, using a versatile material particle generator we have been able to explore the simultaneous influence of size and density on the turbulent transport of material particles. For fixed carrier flow conditions, acceleration statistics of finite size inertial particles are found very robust to size and density variations, the influence of which is mostly carried by acceleration variance only. In particular the shape of acceleration probability density function remains unchanged over the whole range of sizes and densities explored. Concerning acceleration variance, we find that, for heavy particles, finite size effects can be trivially extrapolated neither from the heavy point particle limit nor from the finite size neutrally buoyant case as both of these limits predict 
a monotonic decrease of acceleration variance with increasing inertia, contradictory with the trends measured for finite size heavy particles. Observed finite size and density effects cannot be accounted by simple filtering arguments only based on Stokes number effects as in the point particle case. A sampling scenario, in the spirit of sweep-stick mechanisms seems more adequate to possibly describe the simultaneous influence of density and size. We believe this should stimulate further experimental and numerical investigations of acceleration fields in turbulent flows as well as of inertial particles concentration fields. It is our hope that these measurements will contribute bridging experimental observations and developments of accurate models for the turbulent transport of particles.

Acknowledgements We deeply acknowledge E. Calzavarini, J.-F. Pinton, R. Volk, and Z. Warhaft for fruitful discussions.

\section{References}

1. M.W. Denny, E.K. Nelson, K.S. Mead, Biological Bulletin 203, 275 (2002)

2. M. Boehm, D. Aylor, Atmospheric Environment 39(27), 4841 (2005)

3. M.T. Boehm, D.E. Aylor, E.J. Shields, Journal of Applied Meteorology and Climatology 47(1), 291 (2008)

4. R. Clift, J.R. Grace, M.E. Weber, Bubbles, drops and particles (Academic Press, New York, 1978)

5. R. Mei, Experiments in Fluids 22(1-13) (1996)

6. K.D. Squires, J.K. Eaton, Physics of Fluids 5, 1169 (1991)

7. R.A. Shaw, Annual Review of Fluid Mechanics 35, 183 (2003)

8. M. Pinsky, A. Khain, Journal of Atmospheric Sciences 61(15), 1926 (2004)

9. R. Gatignol, Journal de Mécanique théorique et appliquée 2(2), 143 (1983)

10. M.R. Maxey, J.J. Riley, Physics of Fluids 26(4), 883 (1983)

11. E. Balkovsky, G. Falkovich, A. Fouxon, Physical Review Letters 86(13), 2790 (2001)

12. L. Zaichik, V.M. Alipchenkov, High Temperature 42(3), 426 (2004)

13. J. Bec, L. Biferale, G. Boffetta, A. Celani, M. Cencini, A. Lanotte, S. Musacchio, F. Toschi, Journal of Fluid Mechanics 550, 349 (2006)

14. M. Bini, W.P. Jones, Physics of Fluids 19(3) (2007)

15. J. Jung, K. Yeo, C. Lee, Physical Review E 77(1, Part 2) (2008)

16. G.A. Voth, A. LaPorta, A.M. Crawford, J. Alexander, E. Bodenschatz, Journal of Fluid Mechanics 469, 121 (2002)
17. N. Mordant, J. Delour, E. Léveque, O. Michel, A. Arnéodo, J.F. Pinton, Journal of Statistical Physics 113, 701 (2003)

18. S. Ayyalasomayajula, A. Gylfason, L. Collins, E. Bodenschatz, Z. Warhaft, Physical Review Letters 97, 144507 (2006)

19. S. Ayyalasomayajula, A. Gylfason, Z. Warhaft, Lagrangian Measurements of Fluid and Inertial Particles in Decaying Grid Generated Turbulence, in IUTAM Symposium on Computational Physics and New Perspectives in Turbulence, edited by Y. Kaneda, Nagoya University (Springer, Nagoya, Japan, 2006), Vol. 4, pp. 171-175

20. N.M. Qureshi, M. Bourgoin, C. Baudet, A. Cartellier, Y. Gagne, Physical Review Letters 99, 184502 (2007)

21. R. Volk, E. Calzavarini, G. Verhille, D. Lohse, N. Mordant, J.F. Pinton, F. Toschi, Physica D 237(14-17), 2084 (2008)

22. H. Xu, E. Bodenschatz, Physica D 237, 2095 (2008)

23. N. Mordant, A.M. Crawford, E. Bodenschatz, Physical Review Letters 93, 214501 (2004)

24. P. Gervais, C. Baudet, Y. Gagne, Experiments in Fluids 42(3), 371 (2007)

25. N. Mordant, P. Metz, J.F. Pinton, O. Michel, Review of Scientific Instruments 76(2), 025105 (2005)

26. N. Mordant, J.F. Pinton, O. Michel, Journal of the Acoustical Society of America 112(1), 108 (2002)

27. N. Mordant, A.M. Crawford, E. Bodenschatz, Physica D 193, 245 (2004)

28. R. Volk, N. Mordant, G. Verhille, J.F. Pinton, European Physics Letters 81, 34002 (2008)

29. N.T. Ouellette, H. Xu, M. Bourgoin, E. Bodenschatz, New Journal of Physics 8, 102 (2006)

30. B. Kadoch, W. Bos, K. Schneider, Physical Review Letters 100, 184503 (2008)

31. D.T. Sandwell, Geophysical Research Letters 14(2), 139 (1987)

32. S. Ayyalasomayajula, Z. Warhaft, L.R. Collins, Physics of Fluids 20(9), 095104 (2008)

33. J.L. Lumley, Topics in Physics 12, 290 (1978)

34. L. Chen, S. Goto, J.C. Vassilicos, Journal of Fluid Mechanics 553, 143 (2006)

35. S. Goto, J.C. Vassilicos, Physical Review Letters $\mathbf{1 0 0}(5), 054503$ (2008)

36. A. Aliseda, A. Cartellier, F. Hainaux, J. Lasheras, Journal of Fluid Mechanics 468, 77 (2002)

37. E. Deutsch, O. Simonin, in Turbulence Modification in Multiphase Flow, ASME-FED 110, 35 (1991) 